Proceedings of the "International Workshop on X-Ray and Neutron Scattering Studies of Magnetic Materials," Grenoble, France, 21-23 January 1993. [to be published in Physica A]

BNL- -48610

DE93 010873

\title{
X-Ray and Neutron Scattering Studies of Magnetic Critical Fluctuations in Holmium
}

\author{
T. R. Thurston, ${ }^{(a)}$ G. Helgesen, ${ }^{(a)}$ J. P. Hill, ${ }^{(a, b)}$ \\ Doon Gibbs, ${ }^{(a)}$ B. D. Gaulin, ${ }^{(a, c)}$ and G. Shirane, ${ }^{(a)}$
}

(a) Department of Physics, Brookhaven National Laboratory, Upton, NY 11973

(b) Department of Physics, Massachusets Institute of Technology, Cambridge, MA 02139

(c) Department of Physics, McMaster University, Hamilton, Ontario 4M1, Canada

\begin{abstract}
We describe measurements of the magnetic critical fluctuations of holmium by $x$-ray scattering techniques. The $x$-ray results are compared to those obtained in neutron scattering experiments performed on the same sample.
\end{abstract}

\section{DISCLAIMER}

This report was prepared as an account of work sponsored by an agency of the United States Government. Neither the United States Government nor any agency thereof, nor any of their employees, makes any warranty, express or implied, or assumes any legal liability or responsibility for the accuracy, completeness, or usefulness of any information, apparatus, product, or process disclosed, or represents that its use would not infringe privately owned rights. Reference herein to any specific commercial product, process, or service by trade name, trademark, manufacturer, or otherwise does not necessarily constitute or imply its endorsement, recommendation, or favoring by the United States Government or any agency thereof. The views and opinions of authors expressed herein do not necessarily state or reflect those of the United States Government or any agency thereof.
RECEIVED APR 071993 OSTI 
A number of studies have shown that the order parameter of a phase transition can be directly measured by x-ray magnetic scattering techniques (see for example, Goldman et al., 1987; Thurston et al., 1988; Hill et al., 1991). Until recently, however, there have been no $\mathrm{x}$-ray scattering measurements of the short-ranged critical correlations which occur above a magnetic ordering transition (Thurston et al., 1993). If such measurements are feasible, then the synchrotron-based $x$-ray scattering techniques can extend and complement, the more traditional techniques of neutron magnetic scattering in at least four areas:

1) High $q$-Resolution: Although high $q$-resolution measurements are possible with neutron diffraction, they are rarely made in studies of critical behavior because the signals are relatively weak. Typically, the radial resolution employed in a synchrotron $x$-ray scattering experiment is approximately one order of magnitude finer than that employed in neutron scattering experiments. Thus, it is possible to probe significantly longer length scales in an $x$-ray scattering experiment than are usually probed in neutron scattering experiments. Indeed, with the current and planned high brightness $\mathrm{x}$-ray synchrotron sources, it has become possible to probe ordering phenomena with correlations extending over micron length scales with high accuracy. At the same time, this allows the characterization of magnetic critical fluctuations at temperatures much closer to $T_{c}$.

2) Extinction-Free Scattering: While the relative weakness of the $x$-ray scattering cross-section may present considerable technical difficulty in $x$-ray experiments, it also constitutes an advantage of the technique. As a result of the weak cross-section, these signal rates usually fall within the range of applicability of the Born approximation, so there is no extinction correction. Extinction effects have seriously inhibited neutron $\therefore$ " $1 / 3$ cattering measurements of the exponent $\beta$ for the magnetic order parameter in all studies of rare earths performed to date (see, e.g., B. D. Gaulin et al., 1988).

3) Quasi-Elastic Approximation: The relatively broad energy resolution typical of most $x$-ray scattering measurements (5-10 eV) may also be considered a weakness of $\mathrm{x}$-ray scattering techniques in some studies. However, in $\mathrm{x}$-ray magnetic critical scattering experiments, the poor energy resolution allows the reasonable assumption 
that the energy shifts suffered by the scattered beam (due to inelastic processes involving phonons, magnons, etc.) are entirely integrated over. Thus, the quasi-elastic approximation is exact, which considerably simplifies both the experiments and their subsequent analysis.

4) Critical Charge Scattering Associated with Lattice Modulations: In many magnetic materials, and in particular among the rare earths, a complex of lattice modulations originating from the magnetoelastic coupling may accompany magnetic ordering (see, for example, Gibbs et al., 1986). The critical behavior exhibited by these lattice modulations, gives rise to charge scattering above the transition, which is largely uncharacterized (Bohr et al., 1990). X-ray scattering is well matched to the study of this critical scattering, and offers a whole new arena for the characterization of magnetic structures.

Thus, the possibility of $\mathrm{x}$-ray magnetic critical scattering opens a variety of new directions for the study of magnetic structures and critical behavior, including probing fluctuations over unprecedented length scales and precise characterization of the lineshapes. At the same time, however, this increased sensitivity imposes more stringent requirements on sample quality.

In this note, we describe recent measurements of critical magnetic scattering in the simple spiral antiferromagnet holmium. The details of this work have been described elsewhere (Thurston et al., 1993), and we report only a few highlights here. Below the magnetic ordering transition temperature $T_{c} \sim 131.2 \mathrm{~K}$, the magnetic moments of holmium are confined to the basal, hexagonally close-packed planes and exhibit spiral magnetic order propagating along the $\hat{c}$-axis. The magnetic diffraction pattern consists of pairs of satellites offset from each of the chemical Bragg reflections by $\Delta Q=(0,0, \tau)$, where $\tau$ is proportional to the turn angle per atomic plane (Koehler et al., 1967; Gibbs et al., 1985). The transition at $131.2 \mathrm{~K}$ has been investigated by a number of techniques, including neutron diffraction (Eckert et al., 1976; Brits et al., 1988; Gaulin al., 1988), specific heat (Jayasuriya et al., 1985; Wang et al., 1991), and dilatometry (Tindall et al., 1977). Most of these experiments have indicated that the transition is second order, however, the dilatometry experiments 
suggest that it is weakly first order. Assuming a second order transition, neutron scattering experiments (Eckert et al., 1976; Brits et al., 1988; Gaulin al., 1988) have determined the values of the critical exponents to be $\nu=0.57 \pm 0.04, \gamma=1.14 \pm 0.1$, and $\beta=$ $0.39 \pm 0.04$. A theoretical description of the magnetic ordering transition in holmium remains controversial with two different universality classes proposed for example, the symmetric $O(n)$ with $n=4$, by Bak et al., 1976, and the chiral universality class by Kawamura, 1988. Still other descriptions of the critical behavior have been offered by Barak et al., 1982, and Azaria et al., 1990.

The experiments were performed at Brookhaven National Laboratory in the National Synchrotron Light Source on beamlines X22C and X25, and in the High Flux Beam Reactor on spectrometer $\mathrm{H} 7$. In the $\mathrm{x}$-ray scattering experiments, the incident photon energy was tuned to $8070 \mathrm{eV}$, which corresponds to the dipole maximum of the $L_{I I I}$ absorption edge. There is a large resonant enhancement ( $>$ a factor 50) of the magnetic scattering when the incident photon energy is tuned near an $L$ or $M$ absorption edge, which was essential for the observation of the critical scattering above $T_{c}$ (Gibbs et al., 1988, 1991; Hannon et $a l ., 1988)$. The resolution in the $\mathrm{x}$-ray experiments obtained at the $(0,0,2-\tau)$ reflection, had half-widths (HWHM) of $0.00029 \AA^{-1}, 0.00045 \AA^{-1}$, and $0.0043 \AA^{-1}$ in the transverse, longitudinal, and out-of-scattering-plane directions, respectively. The neutron scattering spectrometer was configured in an energy integrating double-axis mode with an incident neutron energy of $14.7 \mathrm{meV}$. The resolution obtained at the $(0,0,2-\tau)$ reflection was 0.0029 $\AA^{-1}, 0046 \AA^{-1}$, and $0.042 \AA^{-1}$ in the transverse, longitudinal, and out-of-scattering-plane directions, respectively. As may be seen, the $x$-ray resolution is about a factor of ten finer than the neutron resolution in all three directions.

The main results of this paper are presented in Fig. 1, where transverse scans through the magnetic satellite, obtained by $x$-ray and neutron scattering measurements, are plotted for several different temperatures. High resolution $x$-ray scattering results are shown in the left hand column and lower resolution neutron scattering results are shown in the right hand column. The top row shows scans which were taken below $T_{c}$, and illustrate the resolution limits of the two experimental configurations. The qualitative difference in the reciprocal space resolution in these scans is clear. As the temperature is increased (center 
and bottom panels), the peak intensities of both the $x$-ray and neutron scattering data decrease and the half-widths increase. This is indicative of critical magnetic scattering.

The most striking feature of the data is that the peak widths measured by $x$-ray and neutron scattering (after deconvolution with the resolution functions) differ from each other by a factor of ten at comparable reduced temperatures. Thus, the $\mathrm{x}$-ray scattering results reveal fluctuations with a length scale that is about ten times longer than those probed by neutron scattering. This surprising result is shown clearly in Fig.2, where the fitted half-widths of the $\mathrm{x}$-ray and neutron magnetic scattering results plotted versus reduced temperature $\left|T-T_{c}\right| / T_{c}$ are shown on a logarithmic scale. The neutron scattering data (open circles) probe length scales of order $10-100 \AA$, while the $x$-ray scattering data probe length scales of $10^{3}-10^{4} \AA$. The temperature dependence in both cases exhibits power law behavior, but the exponent $\nu$ obtained from the $x$-ray scattering data $(\nu \sim 1.1)$ is almost twice that obtained by neutron scattering $(\nu \sim 0.57)$. It is also seen that the power law behavior determined by neutron scattering breaks down below a reduced temperature of $\sim 10^{-2}$. This is discussed in more detail in Thurston et al., 1993. The data seem to suggest that there are two length scales, instead of one, in the critical fluctuations of holmium near $T_{c}$. This has subsequently been confirmed!

High and low resolution $x$-ray and neutron scattering experiments have now been performed on this sample to investigate the role of the resolution function in determining what is observed by the two scattering techniques. In addition, several samples with different surface finishes have been prepared and studied in order to investigate whether the near-surface structure plays a role in the $x$-ray scattering experiments. (Since the penetration depth of $\mathrm{x}$-rays in holmium at these wavelengths is only $\sim 1 / 2 i$, while neutrons penetrate $\sim 1 / 2 \mathrm{~cm}$, it is possible that the magnetic critical behavior in the "skin" of the sample probed by $x$-ray scattering may differ from that of the bulk.) These measurements gave results similar to those shown in Figs. 1 and 2, and have been described in a separate article (Thurston et al., 1993). We do not repeat them here. It is worth noting, however, that the critical scattering associated with the cubic-to-tetragonal structural transitions of the perovskites also exhibits two components in reciprocal space, similar in many respects to what is observed in holmium (see McMurrow et al., 1990). In the case of the perovskites, 
the longer ranged fluctuations are believed to arise from a random distribution of structural defects, which act as nucleation sites for the tetragonal phase. The present results may therefore be a magnetic analog of the two length scale problem of the perovskites. Indeed, it is possible that these effects are more common in magnetic ordering transitions than was previously realized. For example, a two component lineshape of the magnetic critical scattering has been observed by $\mathrm{x}$-ray scattering in the transuranium compound $N p A s$ (Langridge et al., 1993; Stirling et al., 1993).

With the advent of high brightness synchrotron sources, many new kinds of experiments have become possible using $x$-ray scattering techniques. Among them, x-ray magnetic scattering studies of critical phenomena offer unprecedented $q$-resoluton, which allows the study of ordering over micron length scales, and highly precise determinations of scattering lineshapes. This, in turn, should lead to a more detailed understanding of magnetic critical phenomena. In this paper, we have shown that it is possible to observe $\mathrm{x}$-ray magnetic critical scattering and, indeed, that there are already surprises in even the most straightforward experiments. Current experiments are concerned with erbium and chromium, and with rare earth thin films.

\section{Acknowledgement}

The work at Brookhaven was supported by the Division of Materials Sciences, U.S. Department of Energy under Contract No. DE-AC02-76CH00016. G. H. would like to acknowledge the Research Council of Norway for partial support. The support of NSF Grant No. DMR-90-07825 is also gratefully acknowledged. 


\section{References}

P. Azaria, B. Delamotte, and T. Jolicouer, Phys. Rev. Lett. 64, 3175 (1990).

P. Bak and D. Mukamel, Phys. Rev. B13, 5086 (1976).

Z. Barak and M. B. Walker, Phys. Rev. B25, 1969 (1982).

J. Bohr, D. Gibbs, D. E. Moncton, K. L. D'Amico, Physica A140, 349 (1986).

J. Bohr, D. Gibbs, and K. G. Huang, Phys. Rev. B42, 4322 (1990).

G. H. F. Brits and P. de V. du Plessis, J. Phys. F18, 2659 (1988).

J. Eckert and G. Shirane, Sol. St. Comm. 19, 911 (1976).

B. D. Gaulin, M. Hagen, and H. R. Child, J. De Physique C8, Supp. 12, 327 (1988).

D. Gibbs, J. Bohr, J. D. Axe, D. E. Moncton, K. L. D'Amico, Phys. Rev. B34, 8182 (1986).

D. Gibbs, D. E. Moncton, K. L. D'Amico, J. Bohr, and B. Grier, Phys. Rev. Lett. 55, 234 (1985).

D. Gibbs, D. R. Harshman, E. D. Isaacs, D. B. McWhan, D. Mills, and C. Vettier, Phys. Rev. Lett. 61, 1241 (1988).

D. Gibbs, G. Grübel, D. R. Harshman, E. D. Isaacs, D. B. McWhan, D. Mills, and C. Vettier, Phys. Rev. B43, 5663 (1991).

A. Goldman, K. M. Mohanty, G. Shirane, T. R. Thurston, C. Peters, R. J. Birgeneau, R. L. Greene, and P. M. Horne, Phys. Rev. B36, 5609 (1987).

J. P. Hannon, G. T. Trammell, M. Blume, and D. Gibbs, Phys. Rev. Lett. 61,1245 (1988). 
J. P. Hili, T. R. Thurston, M. Ramstaad, R. J. Birgeneau, and R. Erwin, Phys. Rev. Lett. 66, 3281 (1991).

K. D. Jayasuriya, S. J. Campbell, and A. M. Stewart, Phys. Rev. Lett. 66, 3195 (1991).

H. Kawamura, Phys. Rev. B38, 4916 (1988).

W. C. Koehler, J. W. Cable, H. R. Child, M. K. Wilkinson, and E. O. Wollan, Phys. Rev. B158, 450 (1967).

S. Langridge, W. Stirling, G. H. Lander, D. Spirlet, J. C. Rebizant, and D. Gibbs (unpublished, 1993)

D. F. McMurrow, N. Hamaya, S. Shimomura, Y. Fujii, S. Kishimoto, and H. Iwasaki, Sol. St. Comm. 76, 443 (1990); and references therein.

W. G. Stirling and G. H. Lander, J. Mag. Mag. Mats. (in press, 1993)

T. R. Thurston, C. Peters, R. J. Birgeneau, and P. M. Horn, Phys. Rev. B37, 9559 (1988).

T. R. Thurston, G. A. Helgesen, D. Gibbs, J. P. Hill, B. D. Gaulin, and G. Shirane, (submitted to Phys. Rev. Lett., 1993).

D. A. Tindall, M. O. Stienitz, and M. L. Plumer, J. Phys. F7, L263 (1977).

J. Wang, D. Belanger, and B. D. Gaulin, Phys. Rev. Lett. $\underline{66}, 3195$ (1991). 


\section{Figure Captions}

Figure 1. Transverse $x$-ray and neutron scattering scans taken at the $(0,0,2-\tau)$ magnetic reflections. The scans in the top row were taken for temperatures just below the magnetic ordering temperature $T_{c}$ and represent the resolution of the respective experimental configurations. The center and bottom rows show critical scattering observed at temperatures above the transition. The solid lines represent fits to a Lorentzian plus Lorentzian-squared lineshape discussed in Thurston et al., 1993.

Figure 2. Half-width-at-half-maxima (HWHM) of the x-ray (closed circles) and neutron (open circles) scattering data taken from fits to transverse scans such as those shown in Fig. 1. The solid lines represent fits to a simple power law of the form $H W H M=\kappa_{0}\left[\left(T-T_{c}\right) / T_{c}\right]^{\nu}$. The resolution limits of the two experimental configuations are shown to the right of the data. 

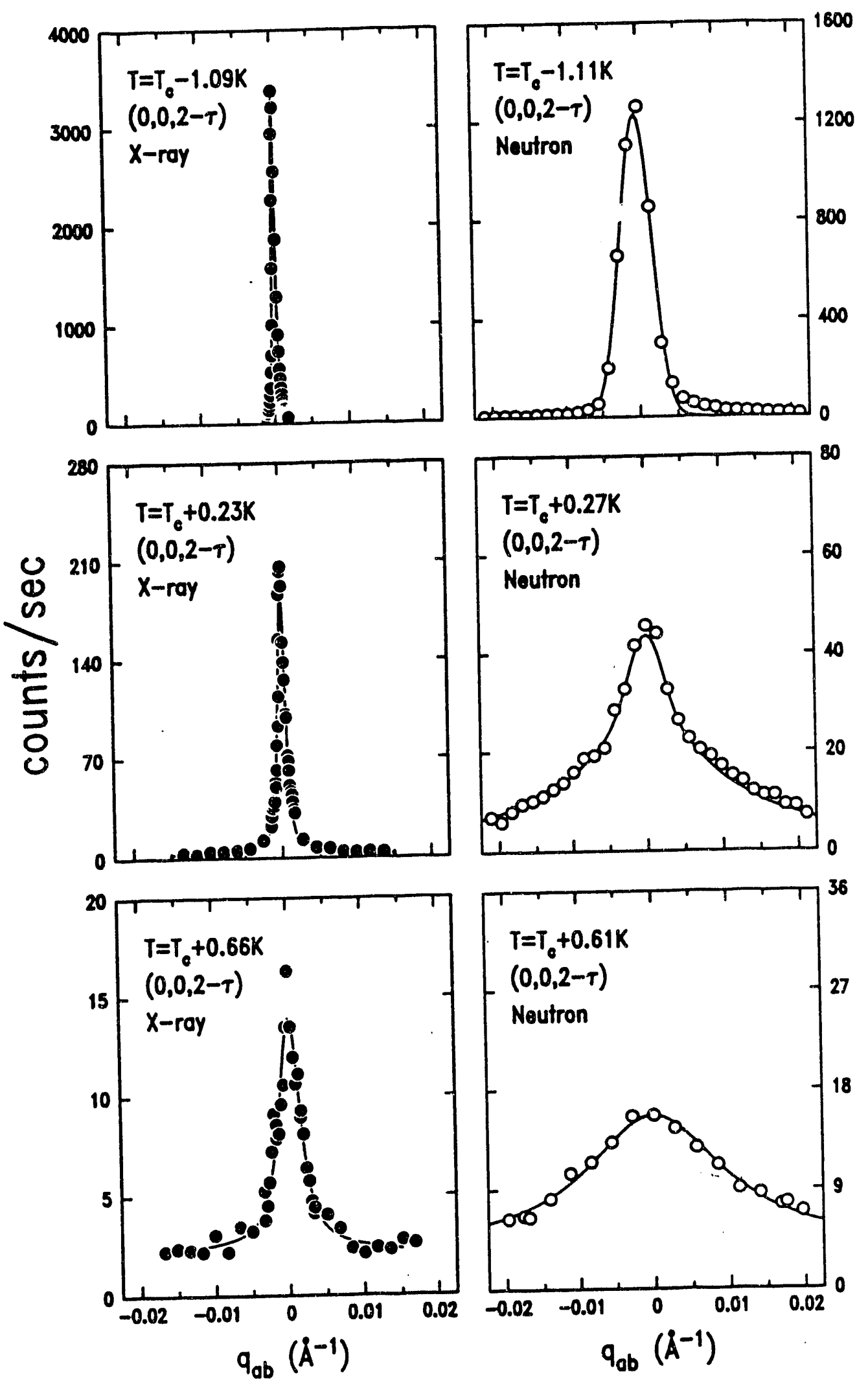


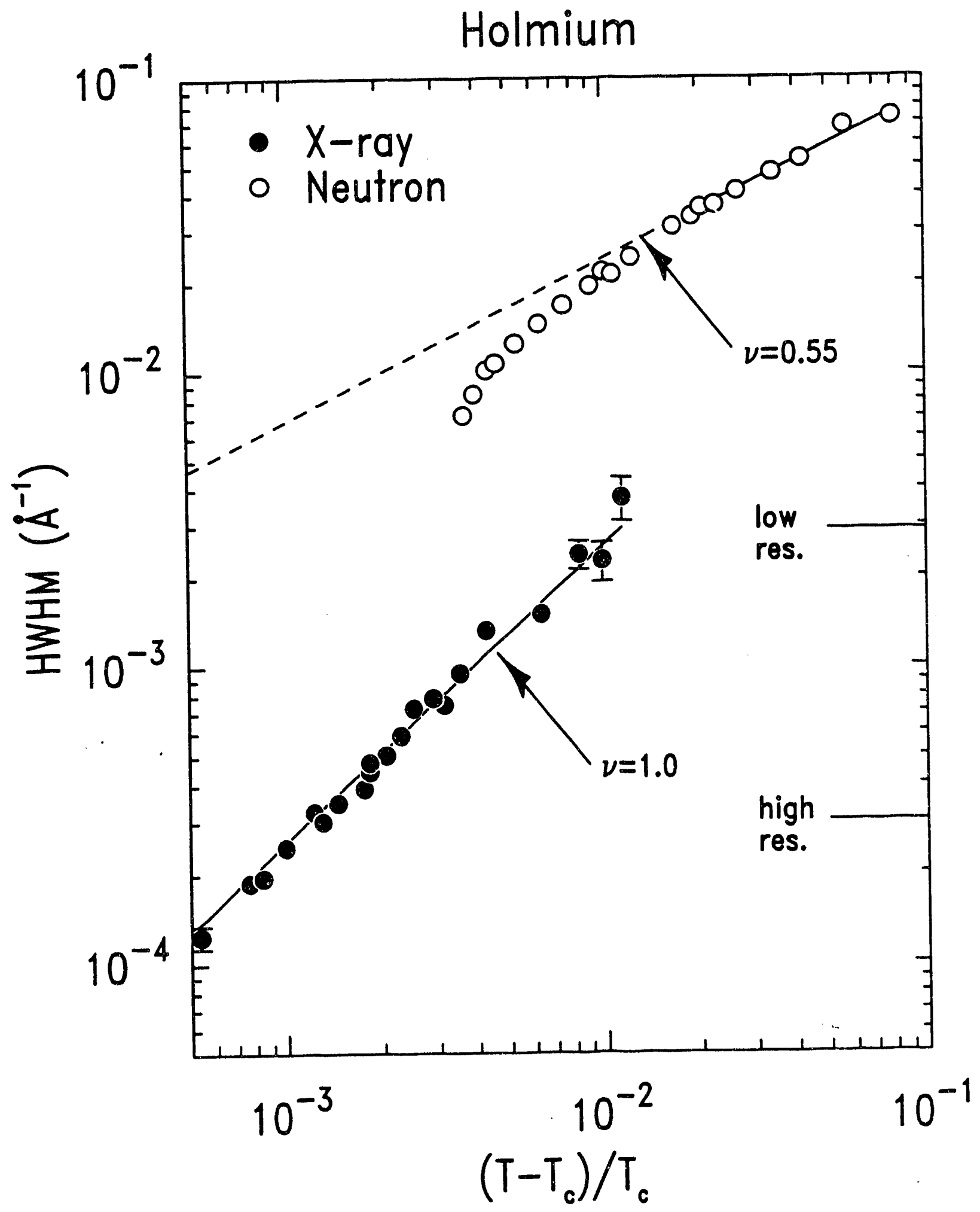



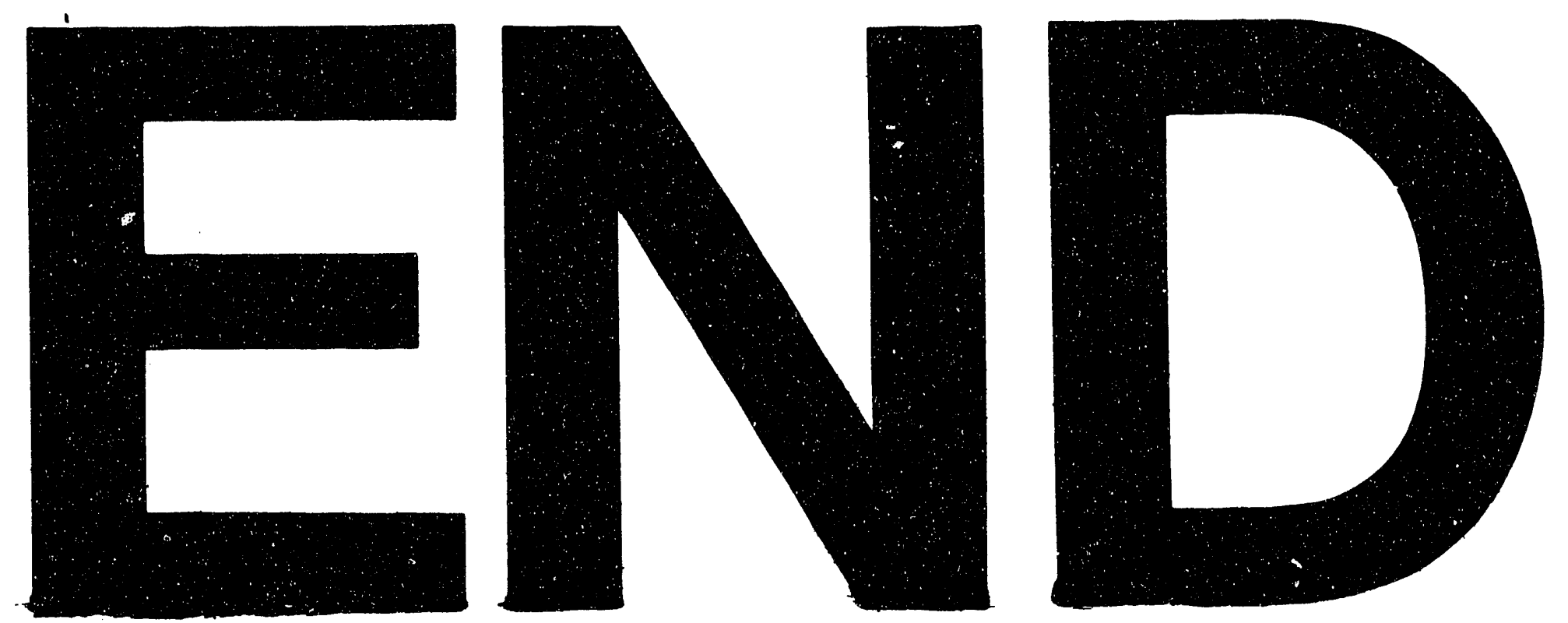

$x$
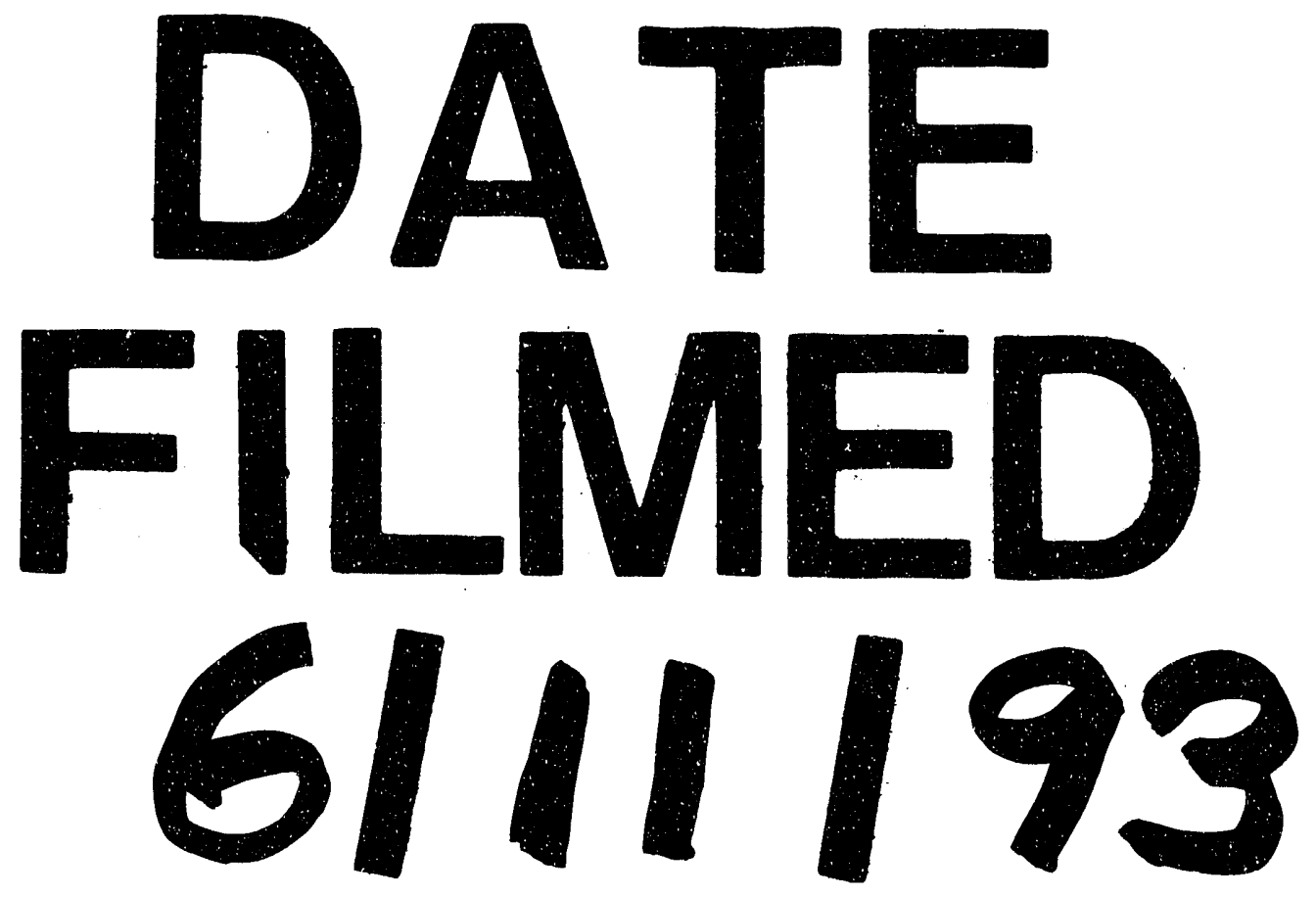